\title{
Impacto de las tecnologías digitales: uso y aplicabilidad en el área médica en tiempos de COVID-19
}

\section{Impact of digital technologies: use and applicability in the medical field in times of COVID-19}

DOI: $10.46932 /$ sfjdv2n2-201

Received in: March 1st, 2021

Accepted in: May 30th, 2021

\section{Biuthy Lisseth Carvajal-Zambrano}

Médica Cirujana; Estudiante de la especialización en Orientación Familiar Integral del Instituto de Posgrado de la Universidad Técnica de Manabí, Portoviejo, Ecuador.

Universidad Técnica de Manabí, Portoviejo, Ecuador

E-mail: biuthycarvajal@gmail.com

\section{Karina Luzdelia Mendoza-Bravo}

Doctora en Ciencias Pedagógicas, Docente Titular de la Universidad Técnica de Manabí, Coordinadora del Departamento de Pedagogía en la Facultad de Filosofía Letras y Ciencias de la Educación,

Coordinadora de la Maestría en Pedagogía, Mención Docencia e Innovación Educativa del Instituto de

Posgrado, Portoviejo, Ecuador.

Universidad Técnica de Manabí, Portoviejo, Ecuador

\section{RESUMEN}

El presente trabajo aborda el rol que han desempeñado las tecnologías digitales en la pandemia sanitaria ocasionada por la COVID-19 en el área médica como primer frente de atención a los pacientes. Tiene por objetivo analizar el impacto de las tecnologías digitales y el uso y aplicabilidad en el área médica en tiempos de COVID-19, para mejorar la calidad del servicio de salud. Para el abordaje metodológico se llevó a cabo una investigación de nivel exploratorio, cuantitativo; donde se empleó como población a los pacientes que acudieron al Hospital Básico Chone por atención médica en el periodo comprendido de marzo hasta abril que fue donde se desarrolló el estudio, aplicando para la muestra un muestreo probabilístico estratificado. Aplicando métodos teóricos, empíricos y estadísticos; dentro de las técnicas encuestas y guías de observación. Se presentan los referentes teóricos más relevantes de las dimensiones analizadas como son el uso de las tecnologías digitales en el área médica, los resultados obtenidos luego del abordaje metodológico con lo cual se pudo establecer el impacto que ha tenido el uso de las tecnologías digitales en el área médica en tiempos de COVID-19, para la mejora de la calidad de servicio de salud. Finalmente se presentan las conclusiones y recomendaciones de este trabajo.

Palabras claves: tecnologías digitales, área médica, COVID-19.

\section{ABSTRACT}

This work addresses the role that digital technologies have played in the health pandemic caused by COVID-19 in the medical area as the first front of care for patients. Its objective is to analyze the impact of digital technologies and the use and applicability in the medical area in times of COVID-19, to improve the quality of health service. For the methodological approach, an exploratory, quantitative research was carried out; where the patients who came to the Chone Basic Hospital for medical attention in the period from March to April, which was where the study was developed, were used as the population, applying a stratified probability sampling for the sample. Applying theoretical, empirical and statistical methods; within the technical surveys and observation guides. The most relevant theoretical references of the 
dimensions analyzed are presented, such as the use of digital technologies in the medical area, the results obtained after the methodological approach, with which it was possible to establish the impact that the use of digital technologies has had on the medical area in times of COVID-19, to improve the quality of health service. Finally, the conclusions and recommendations of this work are presented.

Keywords: digital technologies, medical area, COVID-19.

\section{INTRODUCCIÓN}

En plena crisis pandémica por el nuevo coronavirus, la tecnología puede ser un aliado clave para lograr mejores resultados, tanto para reducir su propagación como para la mitigación y reducción de sus impactos.

La experiencia de países como Corea en el tratamiento de esta emergencia sanitaria demuestra que a través de soluciones tecnológicas como la que describiremos a continuación se puede lograr aplanar la curva de crecimiento del número de personas afectadas con el coronavirus. Este resultado se basa en dos pilares fundamentales: la identificación de las personas afectadas y la separación de estas (distanciamiento social) al igual que las moléculas de aceite se separan de las moléculas de agua.

En una primera etapa resulta fundamental identificar y tener controladas a las personas que entran en el país procedentes de otros destinos afectados o bien ubicar a aquellas personas que actualmente están ubicadas en áreas geográficas dentro de un país que ya están afectadas con el virus. Para lograr esto, es necesaria una coordinación público-privada entre gobiernos y empresas de tecnología, que permita, a partir de soluciones ágiles como las aplicaciones móviles, que los ciudadanos realicen (como parte de las medidas definidas por las autoridades) una autoevaluación de salud a través de preguntas sencillas, similar a lo presentado en la siguiente figura, y tal como lo implementó Corea en el momento que alcanzó los 1.000 casos.

La buena noticia es que algunos países de la región están aprendiendo rápidamente de estas experiencias. Por ejemplo, el 20 de marzo, el gobierno de Uruguay lanzó una aplicación de este tipo y realizó una fuerte campaña de comunicación para promover su difusión y uso. Esta solución supondría, además de la instalación de la aplicación como medida obligatoria a lo largo de los 14 días de cuarentena, contar con un equipo de big data y análisis en el Gobierno, el cual realizará un seguimiento de las estadísticas y llamadas a los ciudadanos que presenten síntomas como parte del ejercicio de autoevaluación. Pero la tecnología, además de apoyar en la fase de prevención, también podría ser un aliado importante en la fase de mitigación pues uno de los grandes desafíos es que las personas sean consistentes durante las próximas semanas en sus acciones de distanciamiento social. 
Dado que los celulares están conectados a antenas móviles que permiten identificar mediante triangulación la ubicación exacta de las personas, estos dispositivos podrían lanzar mensajes de alerta tanto a los ciudadanos potencialmente infectados para que volvieran a la situación de aislamiento bajo implementación de multas o sanciones específicas. Es más, cuando esto ocurriera, los operadores de telefonía móvil podrían enviar mensajes tanto a los servicios de emergencia 911 como a las fuerzas de seguridad para garantizar el aislamiento y el no contagio a otras personas. Es importante tener en cuenta que estas medidas de emergencia sean implementadas dentro de un contexto legal donde los derechos a la privacidad de los ciudadanos sean tomados en consideración para que no ocurran abusos por parte de gobiernos y empresas.

El ejemplo presentado demuestra el potencial de las tecnologías digitales, la conectividad y los datos para el desarrollo socioeconómico y para la gestión de situaciones de crisis. Existe toda una batería de aplicaciones móviles y soluciones digitales en distintas partes del mundo que apoyan diversas áreas de la actividad productiva (agricultura, turismo, energía, logística) y social (salud, educación, gobierno electrónico) que además de contribuir a la reducción de la brecha digital, también contribuyen directamente a la mejora de la calidad de vida de las personas.

En vista de lo manifestado se plantea la siguiente situación problemática en el contexto ecuatoriano se puede indicar que desde que comenzó la pandemia, los servicios de salud de rutina fueron reorganizados o interrumpidos y muchos dejaron de brindar atención a las personas en tratamiento contra enfermedades como el cáncer, enfermedades cardiovasculares y diabetes. Asimismo, muchos trabajadores de la salud que suelen brindar esta atención fueron redirigidos a la respuesta de COVID-19.

La autora de esta investigación al estar inmersa en el área de salud pudo evidenciar que, en el Hospital Básico Chone, también se presentó la problemática, desde el inicio de la emergencia sanitaria se han atendido a más de 2 mil personas con síntomas respiratorios, además, se mantienen los servicios de salud para pacientes no respiratorios, emergencias y cirugías. Para los usuarios con síntomas respiratorios que no requieren hospitalización, el médico realiza la ficha epidemiológica, prescribe el tratamiento y se agenda la cita para la toma de muestras de hisopados. Los usuarios pueden revisar en línea los resultados del examen, para el efecto, deberán digitar el nombre de la unidad médica, número de cédula y el número de la orden. Al inicio de la emergencia sanitaria, el Hospital Básico Chone (HBC) del Instituto Ecuatoriano de Seguridad Social (IESS) disponía de 10 camas para atención a pacientes respiratorios, actualmente esta casa de salud incrementó a 30 el espacio para atención primaria a personas con estas dolencias.

En base a la problemática presentada se formula el siguiente problema: ¿Qué impacto ha tenido el uso de las tecnologías digitales en el área médica y su impacto en tiempos de COVID-19? Y se establece 
como objetivo: Analizar el uso de las tecnologías digitales en el área médica y su impacto en tiempos de COVID-19, para mejorar la calidad del servicio de salud.

Para el abordaje metodológico se llevó a cabo una investigación de nivel exploratorio, cuantitativo; donde se empleó como población a los pacientes que acudieron al Hospital Básico Chone por atención médica en el periodo comprendido de marzo hasta abril que fue donde se desarrolló el estudio, aplicando para la muestra un muestreo probabilístico estratificado.

Este trabajo aborda los referentes teóricos más relevantes de las dimensiones analizadas como son el uso de las tecnologías digitales en el área médica. Las posibilidades que las tecnologías digitales ponen a disposición de la comunidad ayudan a una mejora de la calidad de vida de la misma y el bienestar de las personas, lo que ayuda también a disminuir los desequilibrios y las desigualdades del acceso a los servicios de salud de los ciudadanos, optimización de la relación coste-beneficio, a la vez que favorecen su desarrollo y crecimiento. En definitiva, permiten que el área médica sea más integrada y estar más interconectada.

Se presentan los resultados obtenidos luego del abordaje metodológico con lo cual se pudo establecer el impacto que ha tenido el uso de las tecnologías digitales en el área médica en tiempos de COVID-19, para la mejora de la calidad de servicio de salud. Finalmente se presentan las conclusiones y recomendaciones de este trabajo.

\section{DESARROLLO}

"Las tecnologías digitales están transformado el mundo de los negocios, del trabajo y de la administración pública”, dijo Jim Yong Kim, presidente del Grupo Banco Mundial. "Debemos continuar conectando a todas las personas para que nadie quede a la zaga, porque la pérdida de oportunidades tiene un costo altísimo. Pero para que los dividendos digitales puedan compartirse ampliamente entre todos los segmentos de la sociedad, los países también deben mejorar el clima de negocios, invertir en la educación y la salud de las personas, y promover el buen gobierno”. (Banco Mundial , 2016)

COVID-19, una enfermedad respiratoria previamente desconocida causada por el coronavirus SARS-CoV-2 (Wu, Zhao, Yu, Chen, \& et al., 2020), (Zhu, Zhang, Wang, Li, \& et al, 2020), fue declarada pandemia por la Organización Mundial de la Salud (OMS) el 11 de marzo de 2020, menos de 3 meses después de que se detectaran por primera vez los casos. Con más de 9,8 millones de casos confirmados y más de 495.000 muertes (World Health Organization, 2020) en todo el mundo, existe una grave preocupación por los efectos mundiales de la salud, la sociedad y la economía de este virus, en particular en las poblaciones vulnerables y desfavorecidas, y en los países de ingresos bajos y medianos con sistemas de salud frágiles (Hopman, Allegranzi, \& Mehtar, 2020), (Lloyd-Sherlock, Ebrahim, Geffen, \& McKee, 
2020). En el momento de este escrito, 7.100 millones de personas viven en países que han tenido restricciones viajantes y sociales sustanciales (Connor, 2020).

Al igual que con el control de los brotes y las pandemias que tiene, ante todo, el control de la pandemia COVID-19 se basa en la detección y contención de grupos de infecciones y la interrupción de la transmisión comunitaria para mitigar el impacto en la salud humana. Durante el brote de peste que afectó a la Europa del siglo XIV, se utilizó el aislamiento de las comunidades afectadas y la restricción del movimiento de la población para evitar una mayor propagación (Tognotti, 2013).

Todos los países están obligados por el Reglamento Sanitario Internacional (2005) (World Health Organization, 2016) capacidad básica para garantizar la preparación nacional para los peligros infecciosos que tienen el potencial de propagarse internacionalmente. La investigación y el desarrollo de nuevos métodos y tecnologías para fortalecer estas capacidades básicas a menudo se producen durante los brotes, cuando la innovación es una necesidad absoluta (Heymann, 2017). Durante el brote de síndrome respiratorio agudo grave en 2003, Hong Kong identificó grupos de enfermedades mediante el uso de sistemas de datos electrónicos (Leung, Hedley, Ho, \& et al, 2004). Durante los brotes de ébola en África Occidental en 2014-2016, los datos de los teléfonos móviles se utilizaron para modelar patrones de viaje (Wesolowski, Buckee, Bengtsson, \& et al, 2014), y los dispositivos de secuenciación de mano permitían un rastreo de contactos más eficaz y una mejor comprensión de la dinámica de los brotes (Quick, Loman, Duraffour, \& et al, 2016). Del mismo modo, también se han implementado tecnologías digitales en la pandemia COVID-19 (Ting, Lawrence, Dzau, \& Wong, 2020), (McCall, 2020).

La revolución digital ha transformado muchos aspectos de la vida. En 2019, el 67\% de la población mundial se había suscrito a dispositivos móviles, de los cuales el 65\% eran teléfonos inteligentes, con el crecimiento más rápido en el África subsahariana (GSMA, 2020). En 2019, se descargaron 204 mil millones de aplicaciones (Pérez, 2020), y a partir de enero de 2020, 3.800 millones de personas utilizaron activamente las redes sociales (Kemp, 2020).

Las desigualdades y la brecha digital desde el 2018, la Resolución de la Asamblea Mundial de la Salud sobre Salud Digital reconoció el valor de las tecnologías digitales en el avance de la cobertura sanitaria universal y los Objetivos de Desarrollo Sostenible. Aunque las tendencias se están reduciendo, hoy en día sigue habiendo una brecha digital, y el 51\% de la población mundial no se suscribe a Internet móvil. La falta de acceso a las comunicaciones móviles se observa en los países de ingresos bajos y medianos, aunque las personas con menor situación socioeconómica en los países de ingresos altos también se ven afectadas. El Centro de Investigación Pew reportó grandes disparidades entre las personas de 18 a 29 años de edad y las mayores de 50 años en su acceso a la comunicación móvil. También hay informes de acceso restringido a Internet móvil, como en las zonas de Myanmar, que han dejado a algunas 
poblaciones desconfiadas de la pandemia. Este brote también ha afectado desproporcionadamente a algunas comunidades, como los grupos étnicos negros y minoritarios, más que otros. Por lo tanto, es esencial desarrollar herramientas y mensajes accesibles puede adaptarse a riesgos específicos, idiomas y contextos culturales. (Budd, Miller, \& Manning, 2020)

Fuentes de datos en línea para la detección precoz de enfermedades Los sistemas establecidos de vigilancia de la población suelen basarse en datos relacionados con la salud de los laboratorios, notificaciones de casos diagnosticados por médicos y redes de vigilancia sincrónica. Las redes de vigilancia sincrónica se basan en informes de síntomas clínicos, como "enfermedad similar a la gripe", en lugar de un diagnóstico de laboratorio, procedentes de centros de atención médica primaria y secundaria centinelas seleccionados, que acuerdan proporcionar datos de vigilancia periódicas de todos los casos. Estas fuentes, sin embargo, en última instancia, se pierden casos en los que no se busca atención médica. En el Reino Unido, por ejemplo, donde hasta hace poco sólo los pacientes hospitalizados y los trabajadores sanitarios eran probados rutinariamente para COVID-19, los casos confirmados representan aproximadamente el 4,7\% de los casos sintomáticos de COVID-19 (Russell, Hellewell, Abbott, \& et al, 2020). Identificar casos no detectados ayudaría a dilucidar la magnitud y las características del brote (Heneghan, Brassey, \& Jefferson, 2020).

La identificación temprana y rápida de los casos es fundamental durante una pandemia para el aislamiento de los casos y los contactos adecuados a fin de reducir la propagación y comprender los principales riesgos y modos de transmisión. Las tecnologías digitales pueden complementar la notificación clínica y de laboratorio, mediante el uso de la identificación de casos basada en síntomas y el acceso generalizado a las pruebas y auto pruebas de la comunidad, y con la automatización y aceleración de los informes a las bases de datos de salud pública. La identificación de casos mediante la notificación de síntomas en línea, como se ve en Singapur y el Reino Unido, se utiliza tradicionalmente para la vigilancia, pero ahora ofrece consejos sobre aislamiento y derivaciones a otros servicios sanitarios, como evaluaciones por vídeo y pruebas. Estos servicios pueden implementarse rápidamente, pero deben estar vinculados a la vigilancia de salud pública en curso y a la acción, como el aislamiento de casos y la cuarentena de contactos. Aunque este enfoque es adecuado para personas sintomáticas, las pruebas generalizadas de personas y poblaciones, así como el rastreo de contactos, tienen un papel crucial en la identificación de casos, ya que se estima que el $80 \%$ de los casos de COVID-19 son leves o asintomáticos. Se están instalando sensores, incluidas cámaras termo gráficas y sensores de infrarrojos, para identificar casos potenciales sobre la base de síntomas febriles (por ejemplo, en los aeropuertos). La gran cantidad de resultados falsos positivos y falsos negativos significa que es poco probable que esto 
tenga un efecto sustancial más allá de aumentar la conciencia. También se están explorando tecnologías portátiles para monitorear COVID-19 en poblaciones (Stanford Medicine, 2020).

\section{RESULTADOS}

Para efectos de este trabajo se consideró al personal de auxiliares en enfermería que se encuentran en el área de triaje, que son los primeros que están en contacto con los pacientes y determinan si es o no positivo a COVID-19. A continuación se presentan algunos de los resultados obtenidos:

Se indago al personal de salud para conocer la frecuencia con la que usaban las tecnologías digitales en sus labores, obteniéndose como resultado que de una población de 61 personas encuestadas se puede establecer que el $64 \%$ utilizan las tecnologías digitales durante sus labores varias veces en una jornada laboral. Esto evidencia que las tecnologías son utilizadas con una alta frecuencia durante el desarrollo de sus actividades.

Se investigó sobre el tiempo óptimo para atender a los usuarios utilizando las tecnologías digitales en donde se establece que el 48\% indican que el lapso óptimo para ser atendidos es de 17 a 22 minutos. Como se puede apreciar el periodo se reduce al utilizar las tecnologías digitales.

Se analizó los motivos por el que utilizan las tecnologías digitales en el área médica, donde se obtuvo de resultado que de una población de 61 personas encuestadas se puede establecer que 33 personas que equivalen al 54\% indican que el motivo por el que utilizan las tecnologías digitales es por el pronto diagnostico en el punto de atención y por ende detectar cual es la problemática del paciente.

$\mathrm{Al}$ analizar el argumento de la ética profesional en los trabajadores que utilizan las tecnologías digitales en el área médica, se puede establecer que el 75\% lo considera como muy buena, conociendo que la aplicabilidad de los medios digitales y de innovación permiten mantener y dar un criterio sobre los aspectos importantes de los diagnósticos clínicos.

Se indago al personal de salud para conocer cómo considera usted la comodidad de las instalaciones para la atención de los pacientes en tiempos de Covid-19, de una población de 61 personas encuestadas se puede establecer que el 70\% indican que la comodidad de las instalaciones para la atención en tiempos de Covid-19 es muy buena, es decir, que se mantienen rigurosos procedimientos de prevención tanto del personal de salud como del paciente.

Otras de las interrogantes hacen referencia a como consideran la limpieza y normas de bioseguridad en tiempos de Covid-19, donde un 72\% de los encuestados consideran que son muy buena; es decir que los criterios dentro del área médica en referencia a la limpieza y normas de bioseguridad se cumplen con normalidad y siguiendo los protocolos respectivos. 
Se puede establecer que el $74 \%$ indican que el servicio al utilizar las tecnologías digitales en el área médica es muy bueno, es decir, que es mucho más rápido y se puede llegar a atender a mucho más pacientes en menor tiempo.

De una población de 61 personas encuestadas se puede establecer que el $80 \%$ recomendarían utilizar las tecnologías digitales en el área médica y agilitar el proceso de detectar enfermedades.

\section{CONCLUSIONES}

Una de las principales motivaciones para la aplicación de las tecnologías de la información por el área de salud, se encuentra en la mejor eficiencia en la gestión de este servicio. En la provisión de los cuidados médicos intervienen una multiplicidad de agentes que necesitan comunicarse, compartir e intercambiar información.

La calidad y la viabilidad económica de una asistencia sanitaria tal como demanda de la sociedad actual depende de la incorporación efectiva del uso de las tecnologías digitales. La plena incorporación estas al área de la salud estará suponiendo mejorar la calidad de vida de los ciudadanos, favoreciendo el desarrollo de herramientas en áreas como el pronto diagnóstico, prevención y tratamiento.

La tecnología se debe contemplar como la herramienta y no como el fin en sí misma. El valor está en la capacidad de construir soluciones a problemas prácticos reales haciendo llegar a los ciudadanos mejoras tangibles en la calidad y acceso a los servicios de salud en tiempos de pandemia Covid-19.

\section{RECOMENDACIONES}

Propiciar acuerdos de colaboración que garanticen la continuidad de los servicios críticos de telecomunicaciones y el acceso gratuito a sitios de información y aplicaciones oficiales sobre el COVID19 en el área médica.

Procurar al mismo tiempo la integridad de la infraestructura de telecomunicaciones y la eficiencia en la prestación del servicio para seguir preservando la tutela de derechos fundamentales de los ciudadanos.

Avanzar decididamente hacia políticas estructurales que permitan la implementación de soluciones digitales avanzadas con el objetivo de tutelar la salud y la educación; y promover el desarrollo productivo. 


\section{REFERENCIAS BIBLIOGRÁFICA}

Connor, P. (1 de abril de 2020). Más de nueve de cada diez personas en todo el mundo viven en países con restricciones de viaje en medio de COVID-19. Obtenido de https://www.pewresearch.org/fact$\operatorname{tank} / 2020 / 04 / 01 /$ more-than-nine-in-ten-people-worldwide-live-in-countries-with-travel-restrictionsamid-covid-19/

GSMA. (2020). La economía móvil. Obtenido de https://www.gsma.com/mobileeconomy/

Heneghan, C., Brassey, J., \& Jefferson, T. (6 de Abril de 2020). CEBMThe Centre for Evidence-Based Medicine develops, promotes and disseminates better evidence for healthcare. Recuperado el 19 de Septiembre de 2020, de https://www.cebm.net/covid-19/covid-19-what-proportion-are-asymptomatic/

Heymann, D. (2017). Vigilancia de la salud pública para enfermedades transmisibles: de rígida y estática a flexible e innovadora. NCBI. doi:10.2105/AJPH.2017.303795

Hopman, J., Allegranzi, B., \& Mehtar, S. (16 de Marzo de 2020). Gestión de COVID-19 en países de ingresos bajos y medianos. JAMA Network. doi:10.1001/jama.2020.4169

Kemp, S. (30 de Enero de 2020). Data Reportal. Recuperado el 19 de Septiembre de 2020, de https://datareportal.com/reports/digital-2020-global-digital-overview

Leung , G., Hedley, A., Ho, L.-M., \& et al. (2 de Noviembre de 2004). La epidemiología del síndrome respiratorio agudo grave en la epidemia de Hong Kong de 2003: un análisis de todos los 1755 pacientes. Annals of Internal Medicine. doi:https://doi.org/10.7326/0003-4819-141-9-200411020-00006

Lloyd-Sherlock, P., Ebrahim, S., Geffen, L., \& McKee, M. (2020). Soportar la peor parte del covid-19: las personas mayores en los países de ingresos bajos y medios. doi:https://doi.org/10.1136/bmj.m1052

McCall, B. (01 de Abril de 2020). COVID-19 e inteligencia artificial: proteger a los trabajadores de la salud y frenar la propagación. The Lancet Digital Health, 2. doi:https://doi.org/10.1016/S25897500(20)30054-6

Pérez, S. (Enero de 2020). Tech Crunch. Recuperado el 19 de Septiembre de 2020, de https://techcrunch.com/2020/01/15/app-stores-saw-record-204-billion-app-downloads-in-2019consumer-spend-of-120-billion/

Quick, J., Loman, N., Duraffour, S., \& et al. (2016). Secuenciación del genoma portátil en tiempo real para la vigilancia del ébola. Nature. doi:https://doi.org/10.1038/nature16996

Russell, T., Hellewell, J., Abbott, S., \& et al. (25 de Agosto de 2020). Uso de una relación de letalidad de caso ajustada por retraso para estimar la sub-información. Centro de Modelización Matemática de Enfermedades Infecciosas (CMMID) Repositorio. Recuperado el 19 de Septiembre de 2020, de https://cmmid.github.io/topics/covid19/global_cfr_estimates

Stanford Medicine. (14 de Abril de 2020). Los científicos de Stanford Medicine esperan usar datos de dispositivos portátiles para predecir enfermedades, incluido COVID-19. Recuperado el 22 de Septiembre de 2020, de http://med.stanford.edu/news/all-news/2020/04/wearable-devices-for-predicting-illness.html 
Ting, D., Lawrence, C., Dzau, V., \& Wong, T. (27 de Marzo de 2020). Tecnología digital y COVID-19. Nature Medicine. Recuperado el 19 de Septiembre de 2020, de https://www.nature.com/articles/s41591020-0824-5

Tognotti, E. (Feebrero de 2013). Lecciones de la historia de la cuarentena, de la peste a la gripe A. NCBI. doi:10.3201/eid1902.120312

Wesolowski, A., Buckee, C., Bengtsson, L., \& et al. (29 de Septiembre de 2014). Comentario: Contención del brote de ébola - el potencial y el desafío de los datos de la red móvil. NCBI. doi: 10.1371/currents.outbreaks.0177e7fcf52217b8b634376e2f3efc5e

World Health Organization. (1 de Enero de 2016). Reglamento Sanitario Internacional (2005) Tercera Edición. Recuperado el 19 de Septiembre de 2020, de https://www.who.int/publications/i/item/9789241580496

World Health Organization. (28 de Junio de 2020). Recuperado el 18 de Septiembre de 2020, de https://www.who.int/docs/default-source/coronaviruse/situation-reports/20200628-covid-19-sitrep160.pdf?sfvrsn=2fe1c658_2

Wu, F., Zhao, S., Yu, B., Chen, Y.-M., \& et al. (03 de febrero de 2020). Un nuevo coronavirus asociado con enfermedades respiratorias humanas en China. Nature. doi:https://doi.org/10.1038/s41586-020-20083

Zhu , N., Zhang, D., Wang, W., Li, X., \& et al. (20 de febrero de 2020). Un nuevo coronavirus de pacientes con neumonía en Chona, 2019. The New England Journal of Medicine. doi:10.1056/NEJMoa2001017 